\title{
Overview of Centers for Disease Control and Prevention's Case Investigation of Cervical Cancer Study
}

\author{
Vicki B. Benard, PhD, ${ }^{1}$ April Greek, $\mathrm{PhD},{ }^{2}$ J. Elizabeth Jackson, $\mathrm{PhD},{ }^{2}$ Virginia Senkomago, PhD, \\ Mei-Chin Hsieh, PhD, MSPH, CTR, ${ }^{3}$ Amanda Crosbie, MS, ${ }^{4}$ Georgetta Alverson, CTR, ${ }^{5}$ \\ Antoinette M. Stroup, PhD, ${ }^{6,7}$ Lisa C. Richardson, MD, MPH, and Cheryll C. Thomas, $\mathrm{MSPH}^{1}$
}

\begin{abstract}
Background: Despite advances in cervical cancer screening, a significant number of women in the United States have not received adequate screening. Studies have suggested that approximately half of the women who developed cervical cancer were not adequately screened. The Centers for Disease Control and Prevention (CDC) Case Investigation of Cervical Cancer (CICC) Study took a unique approach to reconstruct the time before a woman's cervical cancer diagnosis and understand the facilitators and barriers to screening and care. This article provides an overview of the study.

Methods: This study included all cervical cancer survivors diagnosed with invasive cervical cancer aged 21 years and older in three U.S. states from 2014-2016. The study design consisted of three different data collection methods, including comprehensive registry data, a mailed survey, and medical chart abstraction. This overview compares the characteristics of cervical cancer survivors in the three states by study participation and eligibility status.

Results: Registries identified 2,748 women diagnosed with invasive cervical cancer. Of these, 1,730 participants were eligible for participation, 28\% $(n=481)$ enrolled in the study and 23\% $(n=400)$ consented to the medical chart abstraction.

Conclusion: The CICC Study is unique in that it addresses, with medical record verification, the medical history of woman 5 years before their cervical cancer diagnosis as well as provides information from the woman on her health care behaviors. This study provides data on a general population of cervical cancer survivors in three states that could be used to guide interventions to increase cervical cancer screening.
\end{abstract}

Keywords: screening, cervical cancer, cancer registry

\section{Introduction}

$\mathbf{E}$ ACH YEAR $\sim 12,900$ women in the United States are diagnosed with cervical cancer, and more than 4,000 die from the disease. ${ }^{1}$ Timely cervical cancer screening allows for the detection and treatment of cervical precancers to prevent progression to invasive cervical cancer. ${ }^{2}$ Cervical cancer incidence and death rates in the United States have reduced by more than half since the 1950's, largely due to the im- plementation of screening with the Papanicolaou (Pap) test. ${ }^{3,4}$ In addition to the Pap test, human Papillomavirus (HPV) screening tests are currently available, offering women more options to get screened. ${ }^{2}$ Despite these advances, a significant number of women (11\%-29\%) in the United States have not received adequate cervical cancer screening ${ }^{5,6}$ and recent national trends have shown that screening may have declined. ${ }^{7}$

Previous studies have suggested that approximately half $(53 \%-56 \%)$ of the women who developed cervical

\footnotetext{
${ }^{1}$ Division of Cancer Prevention and Control, Centers for Disease Control and Prevention, Atlanta, Georgia.

${ }^{2}$ Battelle, Seattle, Washington.

${ }^{3}$ Louisiana Tumor Registry and Epidemiology Program, School of Public Health, Louisiana State University Health Sciences Center, New Orleans, Louisiana.

${ }^{4}$ New Jersey Department of Health, Cancer Epidemiology Services, Trenton, New Jersey.

${ }^{5}$ Michigan Cancer Surveillance Program, Lansing, Michigan.

${ }^{6}$ Rutgers School of Public Health, Piscataway, New Jersey.

${ }^{7}$ Rutgers Cancer Institute of New Jersey (CINJ), New Brunswick, New Jersey.
} 
cancer in the United States were not adequately screened. ${ }^{8,9}$ These studies were conducted more than 10 years ago in managed care health plan settings among women who had access to free or low-cost screening. The findings have not been confirmed more recently by any large studies. To reduce the cervical cancer burden and reach national cervical cancer screening objectives, it is essential to understand the facilitators or barriers to screening for women who are rarely or never screened so that appropriate, tailored interventions can be developed for this population.

The Centers for Disease Control and Prevention (CDC) conducted a retrospective study which aimed to address this research gap among women who are survivors of invasive cervical cancer. This study, also known as the Case Investigation of Cervical Cancer (CICC) Study, took a unique approach to reconstruct the time before a woman's cervical cancer diagnosis and understand the facilitators and barriers to screening and care. CICC aims to answer the following research questions: (1) Did women who were diagnosed with invasive cervical cancer get screened at any time during the 5 years before their cervical cancer diagnosis? (2) What were the facilitators or barriers to getting screened? (3) Did cervical cancer survivors get the recommended follow-up for an abnormal test in a timely manner? (4) What were the facilitators or barriers to getting follow-up for an abnormal test? (5) What were the women's patterns of seeking medical care (i.e., routine medical care or care for symptoms)?

The purpose of this study is to provide an overview of the methods used to construct the CICC Study and discuss its unique challenges.

\section{Methods}

\section{Study participants}

This study included all cervical cancer survivors diagnosed with invasive cervical cancer 21 years and older in three U.S. states; those diagnosed between 2014 and 2016 in Michigan and New Jersey and between 2013 and 2016 in Louisiana.

\section{Study design}

The study design consisted of three different data collection approaches (OMB \#0920-1162).

- Cancer registry data: Data that are already collected on all invasive cervical cancer diagnoses, including demographic and tumor characteristics, were requested by each participating cancer registry. These data were used to compare characteristics by participation and eligibility status.

- Survey: A questionnaire was mailed to cervical cancer survivors. The purpose of the survey was to identify self-reported barriers and facilitators to screening and treatment before diagnosis, and to examine recall of screening tests. Women who completed the survey were considered enrolled in the study.

- Medical chart abstraction: Enrolled cervical cancer survivors were asked to provide consent so that detailed clinical information about all screening and treatment before diagnosis could be obtained. The clinical information also was used to verify the selfreported screening results.

\section{Cancer registry data}

By state legislatives and laws, cancer data must be reported to a central cancer registry. ${ }^{10}$ Cancer reporting is available for the entire U.S. population because of state support of their cancer registries and the combined efforts of federal cancer registry programs supported by the $\mathrm{CDC}^{11}$ and the National Cancer Institute (NCI). ${ }^{12}$ The three selected state cancer registries provided the population of women eligible for this study. Elements that were reported to the central cancer registry and consolidated for a cervical cancer case include the following: date of diagnosis, histology, primary site, behavior, and summary stage of tumor; patient vital status, and characteristics at the time of diagnosis, including age, race, Hispanic ethnicity, marital status, metropolitan residence, census tract poverty indicator, and insurance carrier.

\section{Survey}

The goal of the survey was to understand the woman's perspective of her health care experience before diagnosis. The survey was designed based on other cervical cancer studies. $^{13-17}$ The survey instrument was 13 pages with 27 questions estimated to take 15 minutes to complete. It consisted of five sections, including (1) cervical cancer history (including whether the survivor was diagnosed as the result of a routine examination or was seeking medical care related to symptoms), use of cervical cancer screenings and follow-up for abnormal tests in the 5 years before diagnosis, and barriers and facilitators to screening and any necessary follow-up; (2) health insurance; (3) other medical conditions; (4) respondent demographic characteristics; and (5) interest in cervical selfsampling technology, HPV vaccination of children, and awareness of HPV before diagnosis. The survey also provided opportunities for patients to comment and recount their personal experience of diagnosis outside of the structured questions. The survey was sent by mail in both English and Spanish. Return of the survey served as consent for participation in the study.

\section{Chart abstraction}

The goal of the chart abstraction was to collect comprehensive medical record data for the 5-year period up to the date of invasive cervical cancer diagnosis. Two clinical consultants (oncologists) and cancer registry experts provided the guidance to develop a structured chart abstraction instrument to collect chart data. The medical abstraction form consisted of six sections, including (1) demographic variables; (2) clinical history relevant to cervical cancer; (3) detailed data for cervical cancer screening; (4) detailed data for colposcopies and cervical biopsies; (5) other diagnostic and treatment procedures; and (6) a checklist of relevant symptoms and the earliest date each was reported. The instrument made ample use of comment (i.e., free text) fields for abstractors to record other information they felt was relevant, or to capture procedure and test data that did not fit into the structured form. Signed medical release forms were required for the cancer registry to conduct this portion of the study.

\section{Data collection}

Phase 1: physician and facility contact. Before distributing the survey and other study materials, the cancer registry 
contacted the diagnosing physician to ensure that there were no contraindications or concerns regarding contacting the woman for the study. The cancer registry sent a cover letter and opt-out forms to the physician. All registries had passive consent, where the physician had up to 2 weeks ${ }^{2}$ to contact the cancer registry; otherwise, the registry proceeded with patient contact. One registry had an additional requirement of reaching out to the facility associated with the diagnosis for passive consent before contacting the physician.

Phase 2: distribute study material packet. Once the physician and facility contact period were complete, the cancer registry mailed study packet materials to the potential participants. The mailing included a cover letter that introduced the study, an informational sheet, the survey, a consent form for medical chart abstraction, a form for the sources of health care from which the patient had received care in the last 5 years, and a prepaid self-addressed stamped envelope. Documents translated into Spanish were available for distribution at the discretion of the cancer registry. All attachments were approved by Institutional Review Board at CDC, Battelle, and all participating central cancer registries. Contact information for the registry was provided on all study materials to answer any questions a patient had about participating in the research study.

Phase 3: follow-up. Starting 1 week (or 5 business days) after the initial mailing, the cancer registry conducted two to three follow-up phone calls at varying times of the day and different days of the week with at least one evening call made between the hours of 5-8 pm (Eastern Time in New Jersey and Michigan and Central Time in Louisiana). Three weeks after initial mailing, a second packet was sent if a reply had not been received or no further contact indicated. Starting 1 week after the second mailing (4 weeks after initial mailing), the cancer registry conducted two to three additional followup phone calls at varying times and days. At each phone contact, a woman was offered to complete survey via a phone interview if that was her preference. If a woman could not be reached after 6 weeks, registries conducted a third mailing with outreach by postcard reminder or full study packet at the discretion of the cancer registry. When the materials were received at the cancer registry, a $\$ 25$ incentive was mailed to the participant.

Phase 4: chart abstraction. Most cancer registry staff have collected medical chart data for research studies focused on cancer treatment. Since this study focused on cancer prevention, training was a strong focus of the CICC Study. Study documentation and training materials provided to registries included a brief document outlining the goals of the study and roles and expectations for registries and abstractors; a detailed listing of each item in the chart abstraction instrument, its definition, and where to look for it in the chart; an extensive list of common terms and synonyms with definitions and acronyms; redacted, annotated samples of cytology and pathology reports for cervical cancer screening and diagnostic procedures; and an issue log where questions and problems raised by registry staff were tracked and answered to share lessons learned across facilities. A chart abstraction lead was identified at each registry and was responsible for distributing study documentation to other abstractors and overseeing data collection.
During the data collection period, each registry selected a minimum of 10 cases to conduct reabstraction. Two registries were able to provide de-identified, scanned copies of charts for the clinical consultant to reabstract; the results of the secondary abstraction were reviewed with the abstractors and the consultant in a series of conference calls. The third registry was unable to provide redacted cases, and they conducted and recorded their own secondary abstraction in accordance with study procedures. In addition, monthly conference calls, which included video conferencing when necessary, were held as a group and individually with each registry during the data collection period to discuss progress, resolve issues, and clarify study procedures as needed. Finally, abstractors were encouraged to ask questions by phone or email, as needed.

The CICC Study staff also worked with registries to establish cross-site standards for the collection of medical records. Two registries obtained all records via medical record requests, either to individual providers or in some cases to clearinghouses; the other registry obtained most records via direct access to electronic health records and made additional requests as needed.

In collecting medical records from providers, cancer registries began with the providers listed in the health care sources form completed by each study participant and, as appropriate, the provider identified in the cancer registry record. If the medical records collected from listed providers referred to relevant records for providers not listed (e.g., a surgical pathology report or procedure note), cancer registries made a reasonable effort to contact these additional providers for the needed records.

\section{Results}

Registries identified 2,748 women aged 21 and older who were diagnosed with invasive cervical cancer in the eligible years (Table 1). Of these, $27 \%(n=755)$ were reported to be deceased. An additional 10\% $(n=263)$ were determined to be out of scope for the study, including use in another recent study $(n=81)$; unable to locate a mailing address $(n=68)$; diagnosis, site, or stage determined to be ineligible $(n=39)$; no social security number $(n=19)$; language barrier $(n=17)$; and contacting physician refused $(n=10)$. Of the 1,730 participants who were eligible for participation, $28 \%(n=481)$ enrolled in the study and $23 \%(n=400)$ consented to the medical chart abstraction. The response rate for the survey (overall, 28\%) was slightly higher than for the chart abstraction $(23 \%)$.

Characteristics of the women as reported in the state cancer registry are presented in Table 2 . Women who were enrolled in the study were similar to those who refused to participate by year of diagnosis, age, ethnicity, marital status, poverty indicator, insurance, histology, and stage at diagnosis. There were slight differences in participation by race and residence at diagnosis. A greater proportion of black women and women of other races refused to participate versus enrolled in the study ( $22 \%$ vs. $18 \%$ for black women and $5 \%$ vs. $3 \%$ for women of other races, respectively). In addition, a greater proportion of women in metropolitan areas, refused to participate than to enroll ( $89 \%$ vs. $85 \%$, respectively) unlike women in nonmetropolitan areas who had a smaller proportion refusing to participate versus enroll in the study (11\% vs. 
Table 1. Response Status for Case Investigation of Cervical Cancer (CiCC) Study by State

\begin{tabular}{|c|c|c|c|c|}
\hline & Louisiana & Michigan & New Jersey & Total \\
\hline Total in sampling frame & 846 & 747 & 1155 & 2748 \\
\hline \multicolumn{5}{|l|}{ Out of scope } \\
\hline Deceased & 271 & 126 & 358 & 755 \\
\hline Other $^{\mathrm{a}}$ & 38 & 93 & 132 & 263 \\
\hline \multicolumn{5}{|l|}{ Study participation } \\
\hline Enrolled & 159 & 154 & 168 & 481 \\
\hline Refused: active or passive & 378 & 374 & 497 & 1249 \\
\hline Response rate $^{\mathrm{b}}$ & $30 \%$ & $29 \%$ & $25 \%$ & $28 \%$ \\
\hline \multicolumn{5}{|l|}{ Chart abstraction participation } \\
\hline Enrolled & 156 & 130 & 114 & 400 \\
\hline Refused: active or passive & 381 & 398 & 551 & 1330 \\
\hline Response rate $\mathrm{b}^{\mathrm{b}}$ & $29 \%$ & $25 \%$ & $17 \%$ & $23 \%$ \\
\hline
\end{tabular}

${ }^{a}$ Other ineligibility includes participating in another study, unable to locate mailing address, missing information, diagnosis determined to be ineligible, language barrier, or physician refused.

${ }^{\mathrm{b}}$ Response rate is (no. of enrolled)/(no. of enrolled + no. of refused).

$15 \%$, respectively). In comparison to women enrolled in the study, those who were out of scope of the study because they were deceased had higher proportion of regional and distant stage cases and fewer squamous cell carcinoma cases. A higher proportion of deceased women were older women (>65 years), on Medicare, black, and separated/divorced or widowed, compared to women enrolled in the study.

Facility outreach during the chart abstraction is reported in Table 3. Overall, 572 facilities were contacted for medical record abstraction for 400 women who consented to have chart abstraction completed. These included both hospital and nonhospital (e.g., provider offices, pathology laboratories, and procedure notes) facilities. Overall, on average, 1.7 records were requested for each woman.

\section{Lessons Learned}

As a group, the registries experienced a number of challenges and reported lessons learned related to requesting and accessing/obtaining medical records. They faced a number of barriers to obtaining relevant and complete records for each woman, including incomplete or no information provided on the health care sources form; contact information for providers who had moved; identification of the correct facility for record requests when providers practiced at more than one location; for nonhospital facilities, highly variable request processes and response times from staff; and records sent that did not match the time frame, type of care, or chart elements requested. Some problems experienced by the registries might have been compounded by providers being more accustomed to requests for cancer care than for preventive care. One registry reported that an advance letter mailed to providers explaining the goals of the study was helpful in getting appropriate responses.

While the questionnaire was pilot tested before administering to cervical cancer survivors, the medical records request form was not pilot tested with staff at reporting facilities. Through the course of constructing history of cervical cancer screening up to and including the day of diagnosis, some abstractors found that oncologists frequently maintain records of gynecologic history for their patients even after diagnosis but were unable to include these data since it was not part of the study protocol.

\section{Discussion}

The CICC Study is unique in that it utilized data from multiple sources-the cancer registries, medical records from 5 years before diagnosis, and surveys completed by the survivors-and thus will provide a comprehensive examination of screening history and factors affecting cervical cancer screening in the United States. This report describing the study's aim and methods found that recruitment of cervical cancer survivors for this study was challenging, with overall low participation rates. Nonetheless, the women enrolled in this study had similar characteristics to those of the general population of cervical cancer survivors in these three states between 2013 and 2016; most of the women were white, non-Hispanic, had insurance, and lived in metropolitan areas. In addition, the stage and histology of cervical cancers in women enrolled in this study were similar to those of other cervical cancer survivors in these states; most cancers had localized stage and squamous cell carcinoma histology. Greater proportions of white and nonmetropolitan women enrolled compared to those who refused to participate in the study. The demographic characteristics (age, race, and ethnicity) of women enrolled in this study are also relatively similar to the overall characteristics of women in the United States diagnosed with cervical cancer. ${ }^{1}$

The low participation rate that we found with the CICC Study is similar to participation rates observed in other studies of cervical cancer survivors (13\%-39\%) ${ }^{18-20}$ Previous studies have documented several reasons for nonparticipation in research among cervical cancer survivors, including being too busy, having ongoing illness, not liking research, and feeling that it was too difficult to discuss their cancer. ${ }^{19}$ Studies have also documented the reluctance of cervical cancer survivors to discuss their cancer experience with anyone other than their health care providers due to the individual fear, guilt, stress, anxiety, and responsibility they feel for their cancer. ${ }^{21,22}$ There is considerable stigma associated with cancer and even more so with cervical cancer, because nearly all cervical cancer cases are caused by a sexually transmitted infection, HPV. ${ }^{21-23}$ Survivors have reported feeling that the sexual nature of cervical cancer makes others think negatively about them as irresponsible women. ${ }^{22}$ A study comparing perceptions of cervical versus 
Table 2. Characteristics of Case Investigation of Cervical Cancer (CiCC) Study by Response Status

\begin{tabular}{|c|c|c|c|c|c|c|}
\hline \multirow{2}{*}{$\begin{array}{l}\text { All women diagnosed with } \\
\text { invasive cervical cancer aged } 21 \\
\text { and older in three states }(\mathrm{n}=2748)\end{array}$} & \multirow{2}{*}{$\frac{\begin{array}{c}\text { Enrolled } \\
(\mathrm{n}=481)\end{array}}{\%}$} & \multirow{2}{*}{$\frac{\begin{array}{c}\text { Refused } \\
(\mathrm{n}=1273)\end{array}}{\%}$} & \multirow{2}{*}{$\frac{\begin{array}{c}\text { Out of scope, } \\
\text { deceased }(\mathrm{n}=755)\end{array}}{\%}$} & \multirow{2}{*}{$\frac{\begin{array}{c}\text { Out of scope, } \\
\text { other }^{\mathrm{a}}(\mathrm{n}=263)\end{array}}{\%}$} & \multicolumn{2}{|c|}{ Total } \\
\hline & & & & & $\mathrm{N}$ & $\%$ \\
\hline \multicolumn{7}{|l|}{ Year of diagnosis } \\
\hline 2013 & 6 & 6 & $10^{\mathrm{b}}$ & $3^{b}$ & 190 & 7 \\
\hline 2014 & 27 & 34 & 33 & 20 & 850 & 31 \\
\hline 2015 & 39 & 36 & 34 & 36 & 977 & 36 \\
\hline 2016 & 28 & 25 & 23 & 41 & 715 & 26 \\
\hline \multicolumn{7}{|l|}{ Age at diagnosis } \\
\hline $21-34$ years & 18 & 16 & $7^{\mathrm{b}}$ & $13^{b}$ & 383 & 14 \\
\hline $35-49$ years & 38 & 38 & 24 & 33 & 923 & 34 \\
\hline 50-64 years & 33 & 32 & 35 & 35 & 914 & 33 \\
\hline $65-79$ years & 9 & 12 & 24 & 14 & 404 & 15 \\
\hline 80 or more years & 2 & 2 & 10 & 5 & 124 & 5 \\
\hline \multicolumn{7}{|l|}{ Race } \\
\hline White & 79 & $73^{b}$ & $65^{b}$ & $70^{\mathrm{b}}$ & 1,943 & 72 \\
\hline Black & 18 & 22 & 32 & 19 & 649 & 24 \\
\hline Other & 3 & 5 & 3 & 11 & 123 & 5 \\
\hline \multicolumn{7}{|l|}{ Hispanic ethnicity of any race } \\
\hline Non-Hispanic & 88 & 87 & 90 & $80^{\mathrm{b}}$ & 2,377 & 87 \\
\hline Hispanic & 12 & 13 & 10 & 20 & 351 & 13 \\
\hline \multicolumn{7}{|l|}{ Marital status at diagnosis } \\
\hline Single (never married) & 32 & 34 & $35^{\mathrm{b}}$ & $32^{b}$ & 919 & 34 \\
\hline Married (including common law) & 45 & 40 & 28 & 33 & 1,000 & 37 \\
\hline Separated, divorced, widowed & 17 & 18 & 30 & 22 & 591 & 22 \\
\hline Unknown & 6 & 8 & 8 & 14 & 225 & 8 \\
\hline \multicolumn{7}{|l|}{ Residence at diagnosis } \\
\hline Nonmetropolitan & 15 & $11^{\mathrm{b}}$ & $9^{b}$ & $7^{\mathrm{b}}$ & 292 & 11 \\
\hline Metropolitan & 85 & 89 & 91 & 93 & 2,439 & 89 \\
\hline \multicolumn{7}{|c|}{ Census tract poverty indicator at diagnosis } \\
\hline $0 \%-<5 \%$ poverty & 26 & 24 & 24 & 27 & 656 & 24 \\
\hline $5 \%-<10 \%$ poverty & 18 & 21 & 18 & 16 & 509 & 19 \\
\hline $10 \%-<20 \%$ poverty & 26 & 26 & 26 & 27 & 696 & 26 \\
\hline $20 \%-100 \%$ poverty & 30 & 30 & 33 & 31 & 829 & 31 \\
\hline \multicolumn{7}{|l|}{ Insurance carrier at diagnosis } \\
\hline Not insured & 8 & 8 & $8^{b}$ & $13^{\mathrm{b}}$ & 229 & 8 \\
\hline Private insurance & 41 & 39 & 21 & 33 & 926 & 34 \\
\hline Medicaid & 21 & 18 & 22 & 16 & 543 & 20 \\
\hline Medicare & 11 & 13 & 32 & 16 & 496 & 18 \\
\hline Insurance, not specified & 7 & 6 & 4 & 4 & 153 & 6 \\
\hline Unknown & 12 & 15 & 13 & 18 & 388 & 14 \\
\hline \multicolumn{7}{|l|}{ Histology } \\
\hline Squamous cell carcinoma & 66 & 68 & $63^{b}$ & $64^{\mathrm{b}}$ & 1,800 & 66 \\
\hline Adenocarcinoma & 31 & 27 & 19 & 24 & 689 & 25 \\
\hline Other & 4 & 5 & 18 & 12 & 246 & 9 \\
\hline \multicolumn{7}{|l|}{ Summary stage } \\
\hline Localized & 58 & 54 & $9^{\mathrm{b}}$ & $47^{\mathrm{b}}$ & 1,140 & 42 \\
\hline Regional & 33 & 36 & 40 & 33 & 993 & 36 \\
\hline Distant & 6 & 6 & 42 & 10 & 454 & 17 \\
\hline Unstaged & 2 & 4 & 9 & 9 & 146 & 5 \\
\hline
\end{tabular}

${ }^{\text {a } O t h e r ~ i n e l i g i b i l i t y ~ i n c l u d e s ~ p a r t i c i p a t i n g ~ i n ~ a n o t h e r ~ s t u d y, ~ u n a b l e ~ t o ~ l o c a t e ~ m a i l i n g ~ a d d r e s s, ~ m i s s i n g ~ i n f o r m a t i o n, ~ d i a g n o s i s ~ d e t e r m i n e d ~ t o ~}$ be ineligible, language barrier, or physician refused.

'Chi-square test of difference between "Enrolled" and each other group separately; significant difference, $p<.05$.

ovarian cancer among young adults found that they felt more moral disgust toward cervical cancer patients and were more likely to perceive them as unwise, dishonest, and dirty because their cancer is caused by HPV compared to ovarian cancer whose known cause is a family history of the disease. $^{23}$ The stigma associated with cervical cancer and the ability of survivors to talk about their disease may also be influenced by cultural norms. In a study of African American women in South Carolina, participants explained that while they could talk about other diseases such as diabetes or hypertension, they felt that it was taboo for them to talk about cervical cancer in the black community. ${ }^{24}$ The reasons for 
Table 3. Medical Chart Abstraction for Cancer Investigation of Cervical Cancer (CICC) StUdy By TyPe OF FACILITY

\begin{tabular}{lcccc}
\hline & Louisiana & Michigan & New Jersey & Total \\
\hline No. of facilities records were requested or accessed from & 181 & 221 & 170 & 572 \\
Hospital facilities & 46 & 32 & 43 & 121 \\
Nonhospital facilities & 135 & 189 & 127 & 451 \\
Women's records requested from each facility; mean (range) & $1.8(1-16)$ & $1.4(1-14)$ & $2.1(1-14)$ & $1.7(1-16)$ \\
\hline
\end{tabular}

nonparticipation in research among cervical cancer survivors are varied and complex.

The unique contribution of this study is the focus on preventive care (i.e., before diagnosis) requesting a comprehensive view of the woman's screening and follow-up history 5 years before her cancer diagnosis with medical record verification. The 5-year timeline was chosen because it was the recommended screening interval for women with normal screening results at the time of the study. ${ }^{25}$ As noted in the lessons learned, examining the 5 years before diagnosis was a very intensive part of data collection with many barriers to obtaining relevant and complete screening records for each woman. Most research studies on cancer with chart abstraction involve the period after diagnosis where the oncologist office would be the primary contact. With this study, many different types of nonhospital facilities, including primary care offices, had to be contacted. The study incorporated multiple modes of record abstraction, including having electronic, phone call records sent by mail or by fax, and manually at the facility's physical location. In a time of transition to electronic data collection, data that are not captured in a standard format in the medical chart require a manual, time-intensive process. Other countries with national coverage of health care have the advantage of linking their cancer data with screening histories through pathology databases or other screening registries, which is much more conducive to this type of data collection. ${ }^{26-29}$ However, despite barriers and the intensive work, the abstractors were able to pull 1.5 records on average for the women in the study who had been seen.

\section{Conclusion}

The CICC Study is unique in that it addresses, with medical record verification, the medical history of woman 5 years before their cervical cancer diagnosis. Many studies are either based on self-report only where the frequencies could be over- or underreported or on medical abstraction only where reasons behind why the woman made the decision she did are not provided. This study incorporated both approaches to determine the actual clinical services from the medical records as well as information from the woman to help document the facilitators and barriers. The data provided by this study, particularly on barriers to, and facilitators of, screening in this population of cervical cancer survivors may be useful in prioritizing approaches for interventions to increase screening coverage in rarely or never screened women. Previous studies examining factors affecting cervical cancer screening have been conducted in select populations, mainly immigrants and uninsured women in the United States. ${ }^{30,31}$ This study provides data on a general population of cervical cancer survivors in three states that could be used to guide interventions to increase cervical cancer screening.

\section{Acknowledgments}

This study is supported by Centers for Disease Control and Prevention, Task Orders 200-2015-F-88212 and 200-2016-F90920. The registries also receive support from the Centers for Disease Control and Prevention's National Program of Cancer Registries: New Jersey State Cancer Registry (NJSCR) under cooperative agreement 5U58DP006279-02 awarded to the New Jersey Department of Health \& Senior Services; Louisiana Tumor Registry (LTR) under cooperative agreement 5U58DP003915 awarded to the Louisiana State University Health Sciences Center; and Michigan Cancer Surveillance Program (MCSP) under cooperative agreement NU58DP003921 awarded to the Michigan Department of Health and Human Services. New Jersey cancer incidence data used in this study were collected with support from the National Cancer Institute's Surveillance, Epidemiology, and End Results (SEER) Program under contract HHSN 261201300021I NCI Control No. N01PC-201300021. The collection of State of Louisiana cancer incidence data is also supported by SEER under contract HHSN $261201300016 \mathrm{I}$.

The authors thank cancer registry staff and physician offices who contributed to the project in NJ, LA, and MI as well as the CICC Study Working Group members: Xiao-Cheng Wu, MD, MPH, CTR; Mary O'Flarity, MN, RN; Christina Lefante, MPH; Molly Ariail, MPH; Lauren Maniscalco, MPH; Glenn Copeland, MS (retired); Amy Marquardt, BA; Adrian Botchway, CTR; Cynthia Nunez, BS; Natalia Herman, MPH; Lisa Paddock, PhD; Karen Pawlish, ScD; and, Warner Huh, MD.

\section{Author Disclosure Statement}

The authors have no conflicts of interest to report. The content of this report does not necessarily represent the official position of the U.S. Centers for Disease Control and Prevention.

\section{References}

1. U.S. Cancer Statistics Working Group. U.S. Cancer Statistics Data Visualizations Tool, based on November 2017 submission data (1999-2016): U.S. Department of Health and Human Services, Centers for Disease Control and Prevention and National Cancer Institute; June 2019. Available at: www.cdc.gov/cancer/dataviz Accessed June 9, 2019. 
2. US Preventive Services Task Force. Screening for cervical cancer us preventive services task force recommendation statement. JAMA 2018;320:674-686.

3. Wingo PA, Cardinez CJ, Landis SH, et al. Long-term trends in cancer mortality in the United States, 1930-1998. Cancer 2003;97(S12):3133-3275.

4. Adegoke O, Kulasingam S, Virnig B. Cervical cancer trends in the United States: A 35-year population-based analysis. J Women's Health 2012;21:1031-1037.

5. Benard VB, Thomas CC, King J, Massetti GM, Doria-Rose VP, Saraiya M. Vital signs: Cervical cancer incidence, mortality, and screening-United States, 2007-2012. MMWR Morb Mortal Wkly Rep 2014;63:1004-1009.

6. Cuzick J, Myers O, Hunt WC, et al. A population-based evaluation of cervical screening in the United States: 20082011. Cancer Epidemiol Biomarkers Prev 2014;23:765-773.

7. White A, Thompson TD, White MC, et al. Cancer screening test use-United States, 2015. MMWR Morb Mortal Wkly Rep 2017;66:201.

8. Sung HY, Kearney KA, Miller M, Kinney W, Sawaya GF, Hiatt RA. Papanicolaou smear history and diagnosis of invasive cervical carcinoma among members of a large prepaid health plan. Cancer 2000;88:2283-2289.

9. Leyden WA, Manos MM, Geiger AM, et al. Cervical cancer in women with comprehensive health care access: Attributable factors in the screening process. J Natl Cancer Inst 2005;97:675-683.

10. Coates RJ. Introduction to the summary of notifiable noninfectious conditions and disease outbreaks-United States. MMWR Morb Mortal Wkly Rep 2016;63:1-4.

11. Centers for Disease Control and, Prevention. National Program of Cancer Registries (NPCR). Available at: https:// www.cdc.gov/cancer/npcr/about.htm Accessed March 30, 2019.

12. National, Cancer, Institute. Survaillance, Epidemiology and End Results (SEER) Program. Available at: https://seer .cancer.gov Accessed March 30, 2019.

13. Spence A, Alobaid A, Drouin P, et al. Screening histories and contact with physicians as determinants of cervical cancer risk in Montreal, Quebec. Curr Oncol 2014;21:294.

14. James AS, Campbell MK, Hudson MA. Perceived barriers and benefits to colon cancer screening among African Americans in North Carolina: How does perception relate to screening behavior? Cancer Epidemiol Biomarkers Prev 2002;11:529-534.

15. Berkowitz Z, Hawkins NA, Peipins LA, White MC, Nadel MR. Beliefs, risk perceptions, and gaps in knowledge as barriers to colorectal cancer screening in older adults. J Am Geriatr Soc 2008;56:307-314.

16. Benard VB, Saraiya M, Greek A, et al. Overview of the CDC Cervical Cancer (Cx3) Study: An educational intervention of HPV testing for cervical cancer screening. J Womens Health 2014;23:197-203.

17. Agency for Healthcare Reserach and Quality. Medical expenditure panel survey; access to healthcare 2009. Available at: https://meps.ahrq.gov/survey_comp/hc_survey/ 2012/AC111912.pdf Accessed March 30, 2019.

18. Wenzel L, DeAlba I, Habbal R, et al. Quality of life in long-term cervical cancer survivors. Gynecol Oncol 2005; 97:310-317.
19. Bradley S, Rose S, Lutgendorf S, Costanzo E, Anderson B. Quality of life and mental health in cervical and endometrial cancer survivors. Gynecol Oncol 2006;100:479-486.

20. Greenwald HP, McCorkle R. Sexuality and sexual function in long-term survivors of cervical cancer. J Womens Health (Larchmt) 2008;17:955-963.

21. Ladd IG, Gogoi RP, Bogaczyk TL, Larson SL. Cervical cancer patients' willingness and ability to serve as health care educators to advocate for human papillomavirus vaccine uptake. J Cancer Educ 2018 [Epub ahead of print]; DOI: $10.1007 / \mathrm{s} 13187-018-1348-2$.

22. Dyer KE. 2010 PK New Award: From cancer to sexually transmitted infection: Explorations of social stigma among cervical cancer survivors. Hum Organ 2010;69:321-330.

23. Shepherd MA, Gerend MA. The blame game: Cervical cancer, knowledge of its link to human papillomavirus and stigma. Psychol Health 2014;29:94-109.

24. Bellinger JD, Millegan W, Abdalla AE. "I'm not ashamed to talk on it!": African-American women's decisions about cervical cancer prevention and control in South Carolina. Womens Health Issues 2015;25:120-127.

25. Moyer VA. Screening for cervical cancer: US Preventive Services Task Force recommendation statement. Ann Intern Med 2012;156:880-891.

26. Hammer A, Soegaard V, Maimburg RD, Blaakaer J. Cervical cancer screening history prior to a diagnosis of cervical cancer in Danish women aged 60 years and older-A national cohort study. Cancer Med 2019;8:418-427.

27. Zucchetto A, Franceschi S, Clagnan E, et al. Screening history of women with invasive cervical cancer in northeast Italy. Eur J Obstet Gynecol Reprod Biol 2010;152: 200-204.

28. Ingemann-Hansen $\mathrm{O}$, Lidang $\mathrm{M}$, Niemann $\mathrm{I}$, et al. Screening history of women with cervical cancer: A 6-year study in Aarhus, Denmark. Br J Cancer 2008;98:1292.

29. Nygård JF, Nygård M, Skare GB, Thoresen SØ. Screening histories of women with CIN 2/3 compared with women diagnosed with invasive cervical cancer: A retrospective analysis of the Norwegian Coordinated Cervical Cancer Screening Program. Cancer Causes Control 2005;16:463474.

30. Adunlin G, Cyrus JW, Asare M, Sabik LM. Barriers and facilitators to breast and cervical cancer screening among immigrants in the United States. J Immigr Minor Health 2019;21:606-658.

31. Akinlotan M, Bolin JN, Helduser J, Ojinnaka C, Lichorad A, McClellan D. Cervical cancer screening barriers and risk factor knowledge among uninsured women. J Community Health 2017;42:770-778.

Address correspondence to: Vicki B. Benard, PhD

Division of Cancer Prevention and Control Centers for Disease Control and Prevention 4770 Buford Highway Atlanta, GA 30341

E-mail: vdb9@cdc.gov 